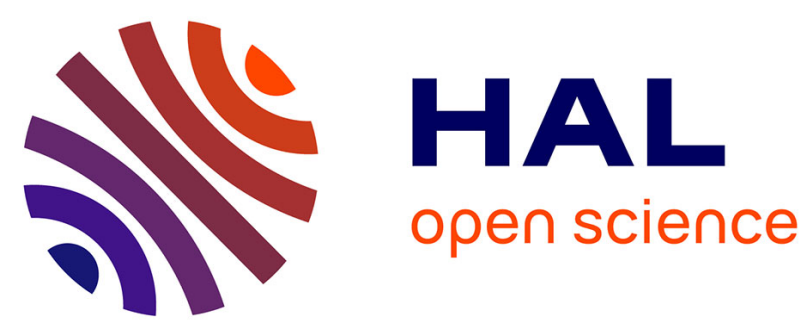

\title{
Recherche d'une distribution de courant produisant une configuration de champ magnétique à symétrie donnée dans un problème à deux dimensions. Application aux lentilles quadrupolaires
}

\author{
J.-C. Mouza
}

\section{To cite this version:}

J.-C. Mouza. Recherche d'une distribution de courant produisant une configuration de champ magnétique à symétrie donnée dans un problème à deux dimensions. Application aux lentilles quadrupolaires. Revue de Physique Appliquée, 1968, 3 (1), pp.83-92. 10.1051/rphysap:019680030108300 . jpa-00242828

\section{HAL Id: jpa-00242828 \\ https://hal.science/jpa-00242828}

Submitted on 1 Jan 1968

HAL is a multi-disciplinary open access archive for the deposit and dissemination of scientific research documents, whether they are published or not. The documents may come from teaching and research institutions in France or abroad, or from public or private research centers.
L'archive ouverte pluridisciplinaire HAL, est destinée au dépôt et à la diffusion de documents scientifiques de niveau recherche, publiés ou non, émanant des établissements d'enseignement et de recherche français ou étrangers, des laboratoires publics ou privés. 


\title{
REGHERGHE D'UNE DISTRIBUTION DE GOURANT PRODUISANT UNE GONFIGURATION DE GHAMP MAGNÉTIQUE A SYMÉTRIE DONNÉE DANS UN PROBLÈME A DEUX DIMENSIONS APPLIGATION AUX LENTILLES QUADRUPOLAIRES
}

\author{
Par J.-C. MOUZA, \\ Institut d'Électronique Fondamentale, Laboratoire associé au C.N.R.S., \\ Bâtiment 220, Faculté des Sciences, 9 I-Orsay. \\ (Reçu le 3 novembre 196\%.)
}

\begin{abstract}
Résumé. - On recherche une distribution de courant produisant une configuration de champ quadrupolaire à gradient constant dans une lentille sans fer à ouverture circulaire. Le problème est abordé pour les systèmes à deux dimensions en supposant les conducteurs infiniment longs. On expose alors une méthode de calcul en coordonnées complexes dont l'exploitation permet de passer en revue les réalisations et les projets actuels, et de donner une formulation générale pour le calcul des harmoniques d'espace.
\end{abstract}

\begin{abstract}
A current distribution is sought for producing a quadrupolar field configuration with constant gradient in an iron-free lens with circular aperture. This problem is studied for the two-dimensional case by assuming the conductors to be infinitely long. A calculation method is expounded in the complex representation, the exploitation of which allows one to examine recent achievements and projects and to give a general formulation of the calculation of space harmonics.
\end{abstract}

Introduction. - Le développement récent de la technique des câbles et rubans supraconducteurs permet d'envisager avec profit le remplacement - particulièrement dans le domaine de l'optique corpusculaire - des électro-aimants classiques par des éléments focalisants supraconducteurs et sans fer. Nous ne nous attarderons pas ici sur les multiples avantages présentés par cette solution et déjà largement développés par ailleurs [1], [2].

Les méthodes de calcul de tels éléments sans fer sont profondément différentes de celles employées avec les électro-aimants à fer doux. Pour un faisceau de particules se déplaçant sensiblement selon un axe $Z^{\prime} Z$, on recherche des répartitions de champ magnétique où le vecteur induction est perpendiculaire aux trajectoires. Sachant qu'en l'absence de pôles ferromagnétiques l'induction magnétique obéit à la seule loi de Biot et Savart, on a alors à résoudre le problème suivant : quelle est la répartition de courants, parallèles à l'axe $Z^{\prime} Z$, qui fournit dans un espace utile donné une distribution idéale de champ transversal, de structure préalablement imposée ? Dans un premier stade, les éléments à calculer sont supposés infiniment longs suivant l'axe $Z^{\prime} Z$; on est ainsi ramené à un problème à deux dimensions car tous les plans perpendiculaires à cet axe sont alors équivalents. C'est ce problème à deux dimensions qui sera abordé ici.
Cette étude sera essentiellement axée sur la recherche de la répartition idéale de champ quadrupolaire. En effet, la focalisation forte de faisceaux de particules chargées, très énergétiques, nécessite l'emploi de lentilles quadrupolaires magnétiques à fort gradient de champ transversal. Les méthodes de calcul proposées sont générales et il est aisé de les transposer à d'autres structures, par exemple aux distributions bipolaires de champ à forts gradients alternés dans les synchrotrons.

Nous rappellerons tout d'abord quelques propriétés des champs, à deux dimensions et exprimés en coordonnées complexes. Pour cela, nous suivrons sensiblement la méthode proposée par R. A. Beth [3] et qui permet d'obtenir l'expression du potentiel-vecteur complexe. Après quoi, nous donnerons à cette formule des extensions nouvelles, dans le but de trouver une répartition de courants, de réalisation aisée au point de vue technologique et fournissant un champ quadrupolaire de structure aussi proche que possible du cas idéal.

Le système d'unités M.K.S.A. rationalisé sera utilisé à l'exclusion de tout autre.

1. Généralités. - 1.1. Potentiel complexe. Considérons un repère orthonormé OXYZ. Le problème étant supposé à deux dimensions, nous nous pla- 
cerons dans un plan $\mathrm{OXY}$ quelconque où tout point $\mathrm{M}$ de coordonnées $X, Y$, peut être représenté par le nombre complexe $\mathscr{Z}=X+i Y=\rho(\cos \theta+i \sin \theta)$. Toutes les lignes de courant considérées ici sont parallèles à $Z^{\prime} Z$ et infiniment longues suivant cet axe. On comptera positivement les courants se déplaçant dans le sens $Z^{\prime} Z$. On suppose que toutes les lignes de courant se referment à l'infini, et ainsi les quantités de courant positives et négatives qui traversent le plan OXY sont égales en module.

Dans l'espace central utile, réservé au faisceau, où ne circule aucun courant, nous pouvons définir un potentiel scalaire $\Phi$. L'induction est donnée par $\mathbf{B}=-\operatorname{grad} \boldsymbol{\Phi}$ et a pour seules composantes $B_{X}$ et $B_{Y}$ :

$$
\begin{aligned}
& B_{X}=-\partial \Phi / \partial X \\
& B_{Y}=-\partial \Phi / \partial Y .
\end{aligned}
$$

Nous pouvons également définir un potentiel vecteur $\mathbf{A}$ ayant une seule composante non nulle : $A_{z}$; on a alors :

$$
\mathbf{B}=\operatorname{rot} \mathbf{A}
$$

soit :

$$
\begin{gathered}
B_{X}=\partial A_{z} / \partial Y \\
B_{Y}=-\partial A_{z} / \partial X
\end{gathered}
$$

Par identification, nous obtenons le système de Cauchy-Riemann :

$$
\begin{aligned}
\partial A_{z} / \partial Y & =-\partial \Phi / \partial X \\
-\partial A_{z} / \partial X & =-\partial \Phi / \partial Y
\end{aligned}
$$

qui montre que la fonction :

$$
\mathscr{A}(\mathscr{Z})=A(X, Y)+i \Phi(X, Y)
$$

est analytique, et exprime en même temps les deux équations fondamentales :

$\operatorname{div} \mathbf{B}=0 \quad$ et $\quad \operatorname{rot} \mathbf{B}=0 \quad$ (dans l'espace utile).

De plus, dans le plan OXY, les deux familles de courbes $A=$ const. et $\Phi=$ const. sont trajectoires orthogonales et définissent respectivement les lignes de force du vecteur induction et les lignes équipotentielles (du potentiel scalaire).

La fonction $\mathscr{A}$ étant analytique, sa dérivée est par définition indépendante de la direction de l'élément d $\mathscr{Z}$ qu'on fait tendre vers zéro, et on calculera aisément :

$$
\begin{aligned}
\beta(\mathscr{Z}) & =\frac{\mathrm{d} \mathscr{A}}{\mathrm{d} \mathscr{Z}}=\frac{\partial A}{\partial X}-i \frac{\partial A}{\partial Y}=\frac{\partial \Phi}{\partial Y}+i \frac{\partial \Phi}{\partial X} \\
& =\frac{\partial A}{\partial X}+i \frac{\partial \Phi}{\partial X}=-B_{Y}-i B_{X} .
\end{aligned}
$$

Il est facile de voir que l'on passe du vecteur $\beta(\mathscr{Z})$ au vecteur $B(\mathscr{Z})$ par la transformation simple :

$$
\left|\begin{array}{l}
B_{X} \\
B_{Y}
\end{array}\right|=\left|\begin{array}{cc}
0 & -1 \\
-1 & 0
\end{array}\right| \cdot\left|\begin{array}{l}
\beta_{X} \\
\beta_{Y}
\end{array}\right|
$$

ou encore :

$$
B(\mathscr{Z})=\overline{i \cdot \beta(\mathscr{Z})}=-i \cdot \overline{\beta(\mathscr{Z})}
$$

le surlignement indiquant que l'on prend le complexe conjugué (soit ici, au total, le produit d'une rotation de $+\pi / 2$ et d'une symétrie par rapport à $\mathrm{OX}$ ).

Le gradient complexe de l'induction est donné par :

$\mathscr{K}(\mathscr{Z})=\frac{\mathrm{d}^{2} \mathscr{A}}{\mathrm{d} \mathscr{Z}^{2}}=\frac{\mathrm{d}}{\mathrm{d} \mathscr{Z}}[\beta(\mathscr{Z})]=-\frac{\partial B_{Y}}{\partial X}-i \frac{\partial B_{X}}{\partial X}$

le premier terme représentant le gradient transversal et le second le gradient longitudinal (au signe près).

1.2. Application au gourant filiforme. - Soit une ligne de courant unique, infiniment longue et perpendiculaire au plan XOY (fig. 1).

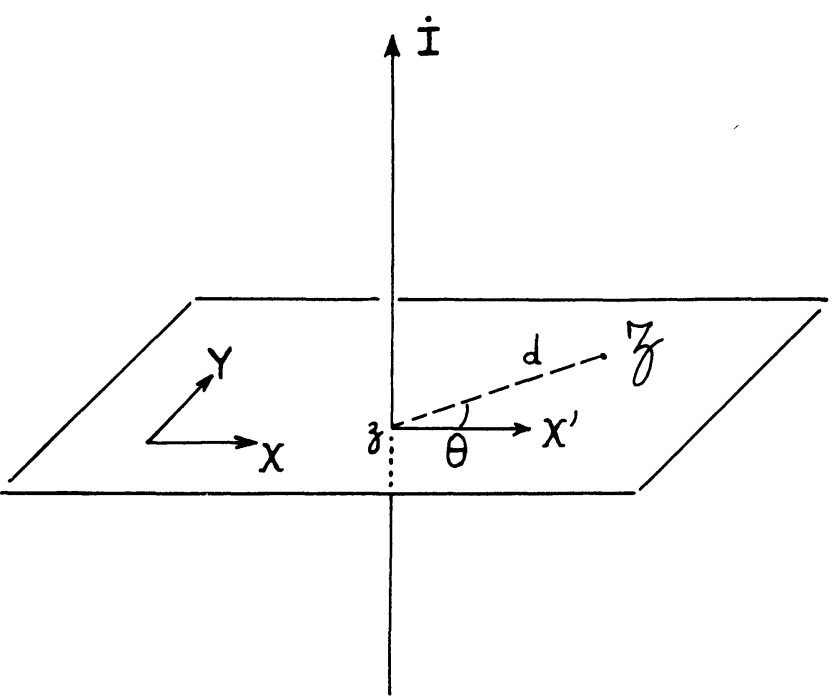

FIG. 1.

Au point d'affixe $\mathscr{Z}$, défini par rapport au point d'affixe $z$ par les coordonnées $(\theta, d)$, on a :

$$
\begin{aligned}
& B_{r}=0 \\
& B_{\theta}=\mu_{0} I / 2 \pi d
\end{aligned}
$$

on peut en déduire le potentiel scalaire multiforme :

$$
\Phi=-\frac{\mu_{0} I}{2 \pi} \theta+\text { const. }
$$

De même, B dérive du potentiel vecteur :

$$
A_{Z}=\frac{\mu_{0}}{4 \pi} I .\left(-\log d^{2}\right) .
$$

Pour définir le potentiel complexe $\mathscr{A}(\mathscr{Z})=A+i \Phi$, remarquons que l'on a :

$\log \mathscr{Z}=\log |\mathscr{Z}|+i \arg \mathscr{Z}$

d'où :

$$
\mathscr{A}(\mathscr{Z})=-\frac{\mu_{0} I}{2 \pi} \log (\mathscr{Z}-z) .
$$


1.3. Appligation a une nappe de gourant. Calculons à présent $\beta(\mathscr{Z})=\frac{\mathrm{d} \mathscr{A}}{\mathrm{d} \mathscr{Z}}=-\frac{\mu_{0} I}{2 \pi} \frac{1}{\mathscr{Z}-z}$ et, dans $\mathrm{OXY}$, considérons une courbe fermée $\mathrm{C}$ entou-

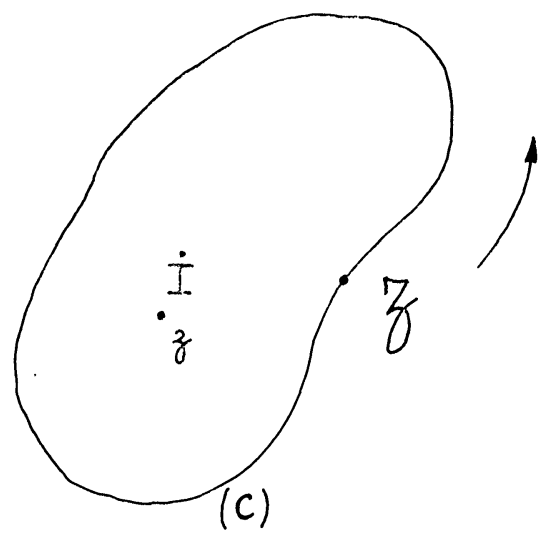

FIG. 2.

rant complètement le point $z$ où passe le courant $I$. La fonction $\beta(\mathscr{Z})$ est holomorphe sur $\mathrm{C}$ et à l'intérieur, sauf en $\mathscr{Z}=z$. Calculons la circulation :

$$
\int_{\mathrm{c}} \beta(\mathscr{Z}) \mathrm{d} \mathscr{Z}=-\frac{\mu_{0} I}{2 \pi} \int_{\mathrm{c}} \frac{\mathrm{d} \mathscr{Z}}{\mathscr{Z}-z}=-i \mu_{0} I
$$

par application de la formule de Cauchy quand on tourne dans le sens positif. C'est là l'expression du théorème d'Ampère dans le plan complexe. En faisant la transformation (2), on retrouverait sa forme classique. (Remarquons que la circulation du vecteur $\beta$ est un imaginaire pur.)

Soit à présent une nappe de courant parallèle à $\mathrm{Z}^{\prime} \mathrm{Z}$ et dont la trace sur OXY est représentée par une courbe $(\Gamma)$. Nous nous proposons de calculer la circulation du vecteur $\beta(\mathscr{Z})$ sur une courbe $(\mathrm{C})$ infi-

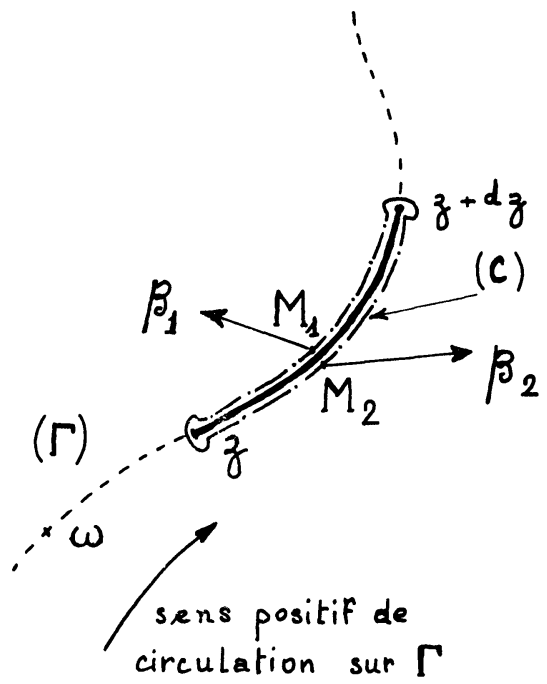

FIG. 3. niment voisine de $(\Gamma)$ et coupant celle-ci aux points d'affixes $z$ et $z+\mathrm{d} z$.

On obtient :

$$
\beta_{2}(z) \mathrm{d} z-\beta_{1}(z) \mathrm{d} z=-i \mu_{0} \mathrm{~d} I
$$

$\mathrm{d} I$ étant la quantité d'électricité passant entre $z$ et $z+\mathrm{d} z$ par unité de temps.

Soit :

$$
\beta_{2}(z)-\beta_{1}(z)=-i \mu_{0} \mathrm{~d} I / \mathrm{d} z
$$

$\mathrm{d} I / \mathrm{d} z$ est la densité linéique de courant au point $z$.

Si on définit sur la courbe $\Gamma$ l'origine $\omega$ des abscisses curvilignes sur cette courbe, on peut intégrer entre l'origine et le point courant $z$ sur $\Gamma$ :

$\int_{\Gamma}\left[\beta_{2}(z)-\beta_{1}(z)\right] \mathrm{d} z=\mathscr{A}_{2}(z)-\mathscr{A}_{1}(z)=-i \mu_{0} I(z)$

à une constante près. S'étant donné une origine $\omega$, on connaît alors en tous points de $\Gamma$ la discontinuité du potentiel complexe $\mathscr{A}$ en fonction de $I(z)$ qui représente la totalité de l'intensité écoulée entre $\omega$ et le point $z$.

2. Induction créée par une répartition de courant sur un cercle. - 2.1. Potentiel gomplexe. - Appliquons la formule (5) à la recherche de l'induction créée par une répartition de courant infiniment mince placée sur un cercle $\Gamma$ centré en $O$.

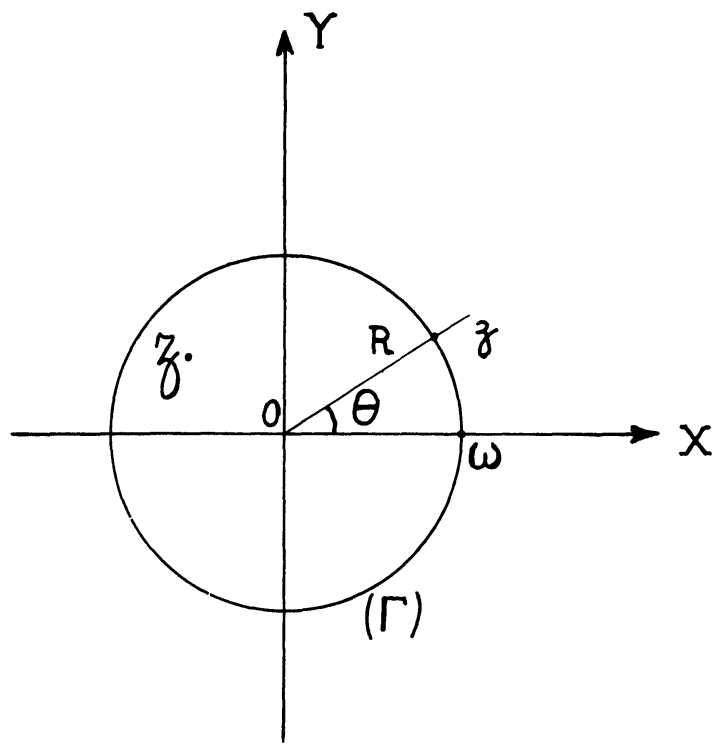

FIG. 4.

Sur le cercle d'équation $z=R \mathrm{e}^{i \theta}$, on $\mathrm{a}$, conformément aux hypothèses déjà précisées : $I(2 \pi)=I(0)=0$.

La fonction $I(\theta)$ étant périodique, elle est décomposable en série de Fourier :

$$
I(\theta)=\sum_{n=1}^{\infty}\left(a_{n} \sin n \theta+b_{n} \cos n \theta\right)
$$


ou :

$$
I(\theta)=\frac{1}{2} \cdot \sum_{n=1}^{\infty}\left[\mathrm{e}^{n i \theta}\left(b_{n}-i a_{n}\right)+\mathrm{e}^{-n i \theta}\left(b_{n}+i a_{n}\right)\right]
$$

dans laquelle les coefficients $a_{n}$ et $b_{n}$ sont homogènes à des intensités; ou encore :

$$
\begin{aligned}
I(\theta)=-\frac{i}{2} \cdot \sum_{n=1}^{\infty}\left[\left(a_{n}+i b_{n}\right)\left(\begin{array}{c}
z \\
R
\end{array}\right)^{n}\right. & \\
& \left.\quad-\left(a_{n}-i b_{n}\right)\left(\frac{R}{z}\right)^{n}\right] .
\end{aligned}
$$

Considérons à présent la formule (5). Nous recherchons les fonctions potentiel complexe $\mathscr{A}_{2}(\mathscr{Z})$ et $\mathscr{A}_{1}(\mathscr{Z})$ respectivement à l'extérieur et à l'intérieur de $(\Gamma)$. Chacune de ces deux fonctions est un potentiel, solution de l'équation de Laplace, pour lequel on connaît les conditions aux limites et qui doit demeurer fini quand $|\mathscr{Z}| \rightarrow 0$ et aussi quand $|\mathscr{Z}| \rightarrow \infty$.

En conséquence, en portant $(6$ bis) dans (5), on obtient :

$$
\begin{aligned}
& \mathscr{A}_{\mathrm{int}}(\mathscr{Z})=\frac{\mu_{0}}{2} \sum_{n=1}^{\infty}\left(a_{n}+i b_{n}\right)\left(\frac{\mathscr{Z}}{R}\right)^{n} \\
& \mathscr{A}_{\mathrm{ext}}(\mathscr{Z})=\frac{\mu_{0}}{2} \sum_{n=1}^{\infty}\left(a_{n}-i b_{n}\right)\left(\frac{R}{\mathscr{Z}}\right)^{n}
\end{aligned}
$$

en prenant $\mathscr{A}$ nul pour $\mathscr{Z}=0$ et $\mathscr{Z}$ infini. Ces deux fonctions qui obéissent aux conditions aux limites et à l'équation (5) sont donc les solutions cherchées; c'est ce système (7) qui, donné par R. A. Beth [3], sert de base aux calculs que nous allons développer ci-dessous.

On pourrait, à partir de ces formules, calculer aisément les vecteurs $\beta(\mathscr{Z})$ et $B(\mathscr{Z})$.

Dans tout ce qui suit, nous noterons :

- $\mathrm{d}^{2} I, \mathrm{~d} I$ ou $I$ les intensités en ampères,

- $J$ les densités linéiques en $\mathrm{A} / \mathrm{m}$,

- $j$ les densités par unité de surface en $\mathrm{A} / \mathrm{m}^{2}$.

2.2. Application : GHAMP QUADRUPolaire IDÉAL GRÉÉ PAR UNE DISTRIBUTION DE GOURANT INFINIMENT MINGE PLAGÉ SUR UN GERGLE. - Utilisant la formule donnant le potentiel complexe à l'intérieur d'un cercle (7), nous allons retrouver la distribution de courant fournissant un champ quadrupolaire idéal [4], [5].

Pour un tel champ, l'intégration de l'équation de Laplace fournit :

$$
\text { et } \quad \begin{aligned}
& \Phi(r, \theta)=B_{2} r^{2} \sin 2 \theta \\
& \quad A(r, \theta)=B_{2} r^{2} \cos 2 \theta \quad \text { pour } r<R .
\end{aligned}
$$

Les lignes équipotentiel scalaire et équipotentiel vecteur constituent deux réseaux d'hyperboles équilatères orthogonales.

On en déduit :

$$
\mathscr{A}=B_{2} \mathscr{Z}^{2} \text { pour } \quad|\mathscr{Z}|<R
$$

d'après (3), on voit que seul subsiste le gradient transversal $K$.

Ce gradient $K$ est donc constant, propriété carac- téristique des champs quadrupolaires et particulièrement recherchée dans les lentilles quadrupolaires afin de réduire au maximum certaines aberrations. Une des qualités d'une lentille réelle pourra donc être caractérisée par la plus ou moins bonne constance de son gradient transversal dans l'espace utile.

Identifions (8) avec la formule (7); soit :

$$
\mathscr{A}_{\text {int }}(\mathscr{Z})=\frac{\mu_{0}}{2} \sum_{n=1}^{\infty}\left(a_{n}+i b_{n}\right)\left(\frac{\mathscr{Z}}{R}\right)^{n} \equiv B_{2} \mathscr{Z}^{2} .
$$

On en déduit aisément, en se reportant dans (6), la répartition de courant sur le cercle, soit :

$$
I=\frac{2 B_{2} R^{2}}{\mu_{0}} \sin 2 \theta=I_{0} \sin 2 \theta
$$

en appelant $\mathrm{d} l$ l'élément de longueur, on a sur le cercle $\mathrm{d} l=R \mathrm{~d} \theta$, ce qui fournit la densité linéique de courant $J$ :

$$
J=\frac{\mathrm{d} I}{\mathrm{~d} l}=\frac{2 I_{0}}{R} \cos 2 \theta \quad(\text { en ampères/mètre }) .
$$

En conséquence, pour réaliser à l'intérieur d'un cercle de rayon $R$ un champ quadrupolaire idéal, il suffit en principe de disposer sur ce cercle une nappe de courant où la densité varie comme $\cos 2 \theta$.

On a déjà réalisé au Brookhaven National Laboratory de tels quadrupoles [6]. Il s'avère que certaines difficultés technologiques ont été rencontrées. En effet, si on désire obtenir un gradient important, il faut réaliser, d'une part, une section conductrice d'épaisseur $\mathrm{d} R$ non négligeable devant $R$, et d'autre part on conçoit facilement l'énorme difficulté à réaliser la variation sinusoïdale de la densité de courant avec l'azimut (un artifice consiste à glisser des cales d'épaisseur variable entre les différents conducteurs).

Il semble difficile, dans ces conditions, d'atteindre un gradient variant de moins de $1 \%$ dans l'espace utile. Aucun résultat précisant la constance du gradient mesurée dans l'espace utile d'un tel type de lentille n'a été publié à ce jour.

3. Gradient fourni par une répartition de courant formant une couche circulaire non infiniment mince. - 3.1. Modification Des fORmules (7). - Considérons à présent une répartition de courant sur une couche circulaire non infiniment mince, d'épaisseur $\mathrm{d} R$. Soit $j$ la densité de courant en $\mathrm{A} / \mathrm{m}^{2}$ dans cette couche conductrice; on a :

$$
\mathrm{d} I(\theta)=\mathrm{d} R \cdot \int_{0}^{\theta} R j(\theta) \mathrm{d} \theta .
$$

La formule (6) est remplacée par :

$$
\mathrm{d} I(\theta)=\sum_{n=1}^{\infty}\left(A_{n} \sin n \theta+B_{n} \cos n \theta\right) \cdot \mathrm{d} R
$$

les coefficients $A_{n}$ et $B_{n}$ étant exprimés en A/m (densités linéiques). 
La contribution au potentiel complexe de la couche d'épaisseur $\mathrm{d} R$, autour de la position moyenne $R$, est :

$\mathrm{d} \mathscr{A}_{\text {int }}(\mathscr{Z})=\frac{\mu_{0}}{2} \sum_{n=1}^{\infty}\left(A_{n}+i B_{n}\right) \cdot\left(\frac{\mathscr{Z}}{R}\right)^{n} \cdot \mathrm{d} R$.

$\mathrm{Si}$ on cherche un champ quadrupolaire idéal, le seul terme non nul est $A_{2}$ et on a toujours $\mathrm{d} I=I_{0} \sin 2 \theta$ avec $I_{0}=A_{2} \mathrm{~d} R$, ou encore :

$$
j(\theta)=j_{0} \cos 2 \theta
$$

avec la relation :

$$
A_{2}=\frac{R j_{0}}{2}
$$

On en déduit :

$$
\mathrm{d} \mathscr{A}_{\text {int }}(\mathscr{Z})=\frac{\mu_{0} j_{0} \mathscr{Z}^{2}}{4 R} \cdot \mathrm{d} R .
$$

Si $j_{0}$ ne dépend pas de $R$, on obtient pour une couche circulaire d'épaisseur $R_{2}-R_{1}$ :

$$
\mathscr{A}_{\text {int }}(\mathscr{Z})=\frac{\mu_{0} j_{0}}{4} \mathscr{Z}^{2} \log \frac{R_{2}}{R_{1}}
$$

le gradient transverse est donné par :

$$
K=-\frac{\mu_{0} j_{0}}{2} \log \frac{R_{2}}{R_{1}} .
$$

Mais comme il est technologiquement peu commode de réaliser des bobinages à densité variable, il semble plus intéressant de rechercher des structures s'approchant au maximum du cas idéal, avec une densité de courant constante dans tout le bobinage.

3.2. Gradient produit Par Des courants DE DeNSITÉ GONSTANTE RÉPARTIS SUR UNE COUGHE GIRCULAIRE ÉPAISSE. - Nous nous proposons de calculer le potentiel $\mathscr{A}$ et le gradient transversal $K$ produits par la structure à 4 bobines schématisée sur la figure 5 , où la densité de courant $j$ est supposée constante dans tout le bobinage. Cette structure présentant, sur un cercle de rayon $R$, une répartition de $j$ en créneaux, a déjà été proposée [7], [8]. Nous donnons ici une méthode de calcul en coordonnées complexes.

La répartition de la densité de courant $j$ sur le cercle de rayon $R$ et d'épaisseur $\mathrm{d} R$ est périodique et est représentée sur la figure $6 \mathrm{~b}$. On a évidemment :

$$
j=\frac{1}{R} \frac{\mathrm{d}^{2} I}{\mathrm{~d} \theta \mathrm{d} R} .
$$

Développons $j$ en série de Fourier à partir de la formule (10). On a :

$$
j(\theta)=\sum_{n=1}^{\infty}\left(\frac{n}{R}\left[A_{n} \cos n \theta-B_{n} \sin n \theta\right]\right)
$$

et calculons les différents coefficients, soit :

$$
-\frac{n}{R} B_{n}=\frac{1}{\pi} \int_{0}^{2 \pi} j(\theta) \sin n \theta \mathrm{d} \theta=0
$$

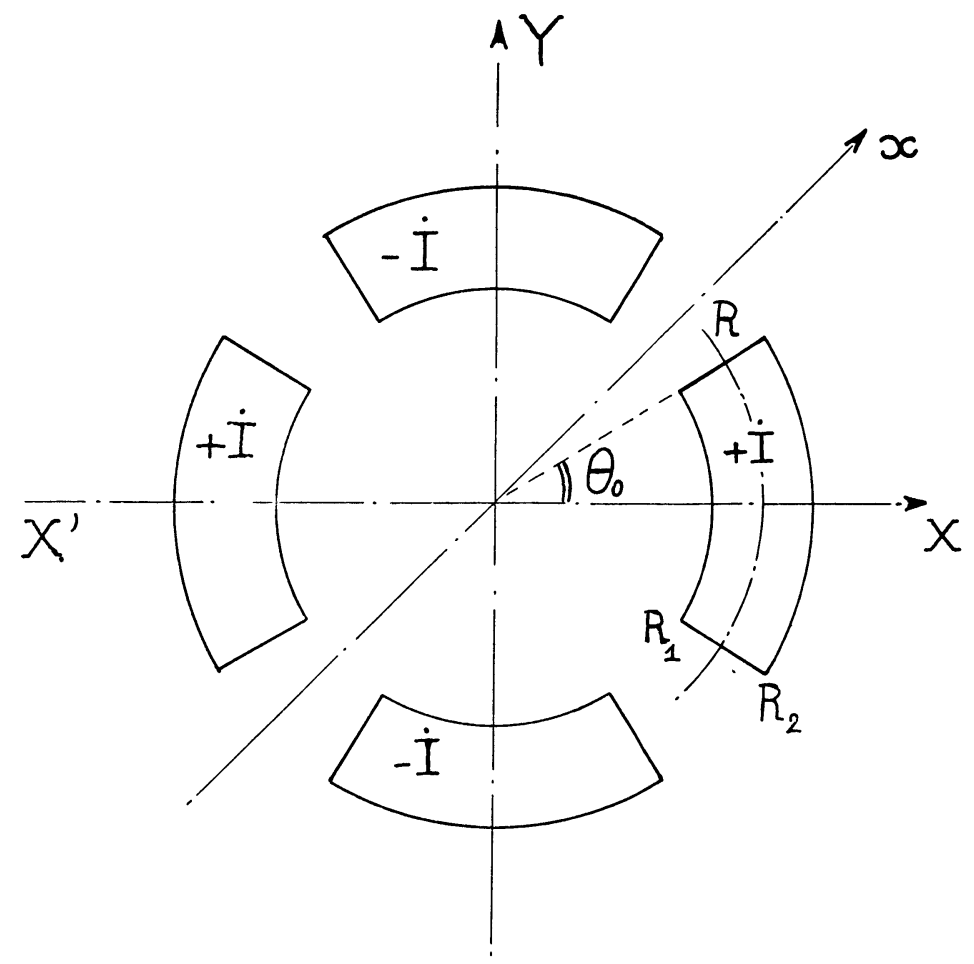

FIG. 5. 


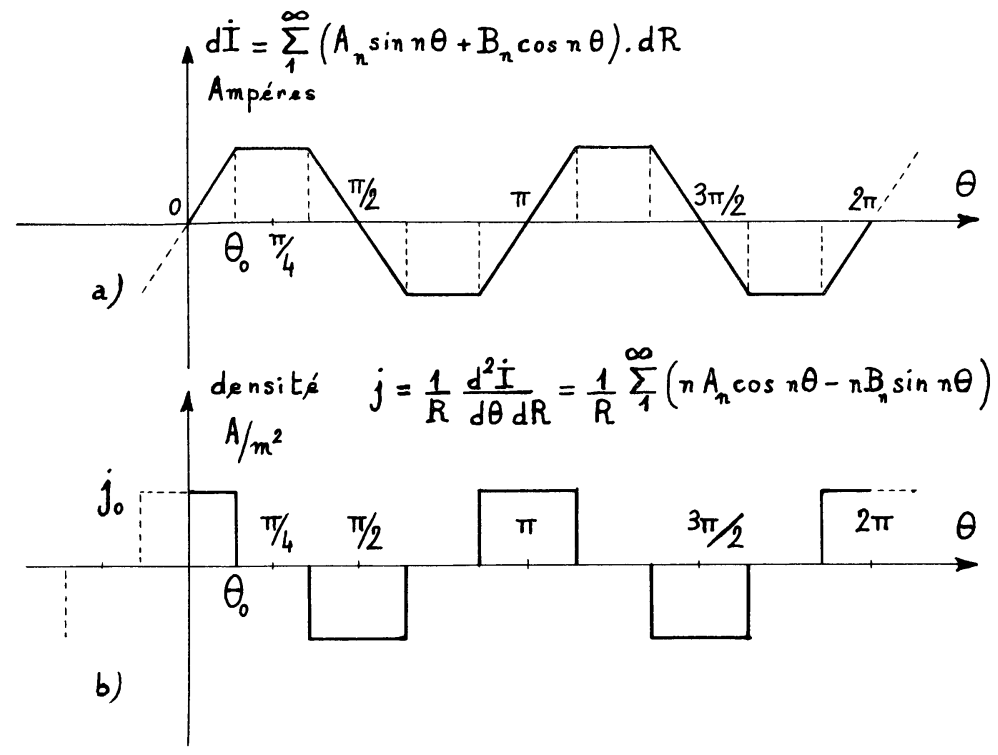

FIG. 6.

et :

$$
\frac{n}{R} A_{n}=\frac{1}{\pi} \int_{0}^{2 \pi} j(\theta) \cos n \theta \mathrm{d} \theta=j_{0} \frac{8 \sin n \theta_{0}}{n \pi}
$$

en appelant ici $j_{0}$ la valeur constante et non nulle de $j$, et $\theta_{0}$ étant défini sur la figure 5 .

D'où :

$$
A_{n}=8 R j_{0} \sin n \theta_{0} / \pi n^{2}
$$

avec :

$$
n=2(2 k+1)
$$

Soit donc :

$$
j(\theta)=\sum_{k=0}^{\infty} \frac{8 j_{0} \sin n \theta_{0}}{\pi n} \cos n \theta .
$$

Or, dans la formule (10), intervient le développement de $I(\theta)$, fonction représentée sur la figure 6 a.

Soit :

$$
\begin{aligned}
& \mathrm{d} I(\theta)=R \mathrm{~d} R \int_{0}^{\theta} \sum_{k=0}^{\infty} \frac{n}{R} \frac{8 R j_{0} \sin n \theta_{0}}{\pi n^{2}} \cos n \theta \mathrm{d} \theta \\
& \mathrm{d} I(\theta)=R \mathrm{~d} R \sum_{k=0}^{\infty} \frac{8 j_{0} \sin n \theta_{0}}{\pi n^{2}} \sin n \theta .
\end{aligned}
$$

Reportant dans (11), on obtient :

$$
\mathrm{d} \mathscr{A}_{\text {int }}(\mathscr{Z})=\frac{\mu_{0}}{2} \mathrm{~d} R \sum_{k=0}^{\infty} \frac{8 j_{0} \sin n \theta_{0}}{\pi n^{2}} \frac{\mathscr{Z}^{n}}{R^{n-1}} .
$$

Intégrons à présent ce résultat pour une couche d'épaisseur $R_{2}-R_{1}$. Soit :

$$
\begin{aligned}
\mathscr{A}_{\text {int }}(\mathscr{Z})=\frac{4 \mu_{0} j_{0}}{\pi} & {\left[\frac{\mathscr{Z}^{2} \sin 2 \theta_{0}}{4} \log \frac{R_{2}}{R_{1}}\right.} \\
& \left.-\sum_{k=1}^{\infty} \frac{\mathscr{Z}^{n} \sin n \theta_{0}}{n^{2}(n-2)}\left(\frac{1}{R_{2}^{n-2}}-\frac{1}{R_{1}^{n-2}}\right)\right]
\end{aligned}
$$

le gradient de l'induction est donné par :

$$
\begin{aligned}
& \mathscr{K}(\mathscr{Z})=\frac{4 \mu_{0} j_{0}}{\pi}\left[\frac{\sin 2 \theta_{0}}{2} \log \frac{R_{2}}{R_{1}}\right. \\
&-\left.\sum_{k=1}^{\infty} \frac{(n-1) \mathscr{Z}^{n-2} \sin n \theta_{0}}{n(n-2)}\left(\frac{1}{R_{2}^{n-2}}-\frac{1}{R_{1}^{n-2}}\right)\right] \\
& \quad \text { avec } \quad n=2(2 k+1) .
\end{aligned}
$$

Sur l'axe OX, le gradient transversal est donné par le développement :

$$
\begin{aligned}
\left(K_{Y}\right)_{\text {ox }}=-\frac{4 \mu_{0} j_{0}}{\pi}\left[\frac{\sin 2 \theta_{0}}{2}\right. & \log \frac{R_{2}}{R_{1}}+\frac{5 X^{4} \sin 6 \theta_{0}}{24}\left(\frac{1}{R_{1}^{4}}-\frac{1}{R_{2}^{4}}\right) \\
& \left.+\frac{9 X^{8} \sin 10 \theta_{0}}{80}\left(\frac{1}{R_{1}^{8}}-\frac{1}{R_{2}^{8}}\right)+\frac{13 X^{12} \sin 14 \theta_{0}}{168}\left(\frac{1}{R_{1}^{12}}-\frac{1}{R_{2}^{12}}\right)+\ldots\right] .
\end{aligned}
$$

Ce résultat a déjà été obtenu par intégration directe [8]. Remarquons qu'avec cette méthode il est aisé, à partir de la formule du gradient complexe, d'atteindre le gradient réel, sur l'axe Ox, se déduisant de OX par une rotation de $+\pi / 4$.
Remarque : En choisissant $\theta_{0}=\pi / 6$, on annule le terme en $X^{4}$. Il est en outre possible de juxtaposer deux couches cylindriques d'épaisseurs respectives $R_{2}-R_{1}$ et $R_{1}-R_{0}$, et d'ouvertures $\theta_{0}$ et $\theta_{0}^{\prime}$ différentes. Par combinaison de tous ces paramètres, on 
peut rechercher la meilleure qualité possible du gradient dans l'espace utile, et s'approcher au maximum de la répartition quadrupolaire idéale.

Une lentille de ce type est en cours de réalisation [9]. Il est certain que cette structure offre, par rapport au modèle de Brookhaven [6] cité ci-dessus, l'intérêt d'être conçue avec une densité de conducteurs constante dans le bobinage. Mais il est difficile de se prononcer sur ses qualités tant que des mesures donnant la répartition du gradient dans l'espace utile n'auront pas été effectuées.

4. Champ quadrupolaire idéal créé par une nappe de courant infiniment mince non circulaire. - Une nappe de courant circulaire, où la densité varie selon la loi donnée par la formule (9), fournit une répartition de champ quadrupolaire idéale. Dans les éléments de longueur $\mathrm{d} l=R \mathrm{~d} \alpha$ situés en $\theta_{1}=\frac{\pi}{4}+N \frac{\pi}{2}$, la densité de courant est nulle. On conçoit intuitivement que des éléments de courants parcourus par des intensités finies, mais placés à l'infini dans les directions $\theta_{1}$ puissent avoir une contribution nulle, comme celle des éléments précédents situés à la distance $R$. On a donc recherché s'il était possible de remplacer la nappe circulaire par une nappe à branches infinies, où la densité linéique $\mathrm{d} I / \mathrm{d} l$ resterait constante.

Nous allons tout d'abord calculer le potentiel complexe créé par 8 petits éléments de courant (traces de 8 fils) situés sur un même cercle $R$ comme l'indique la figure 7 .

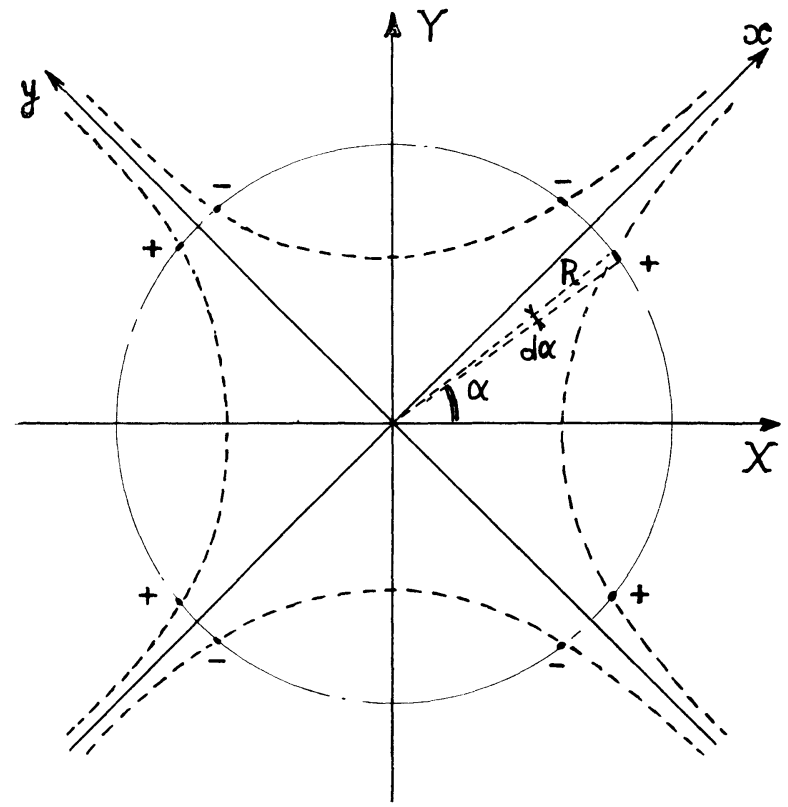

FIG. 7.

Après quoi nous chercherons à intégrer ce résultat sur des courbes ayant la symétrie quadrupolaire et les axes $\mathrm{Ox}$ et $\mathrm{Oy}$ pour directions asymptotiques.
4.1. Potentiel produit par 8 fils. - Sur le cercle de rayon $R$, la distribution de courant correspondant est représentée en fonction de $\theta$ sur la figure $8 \mathrm{c}$. Soit $J_{0}$ la densité de courant linéique.
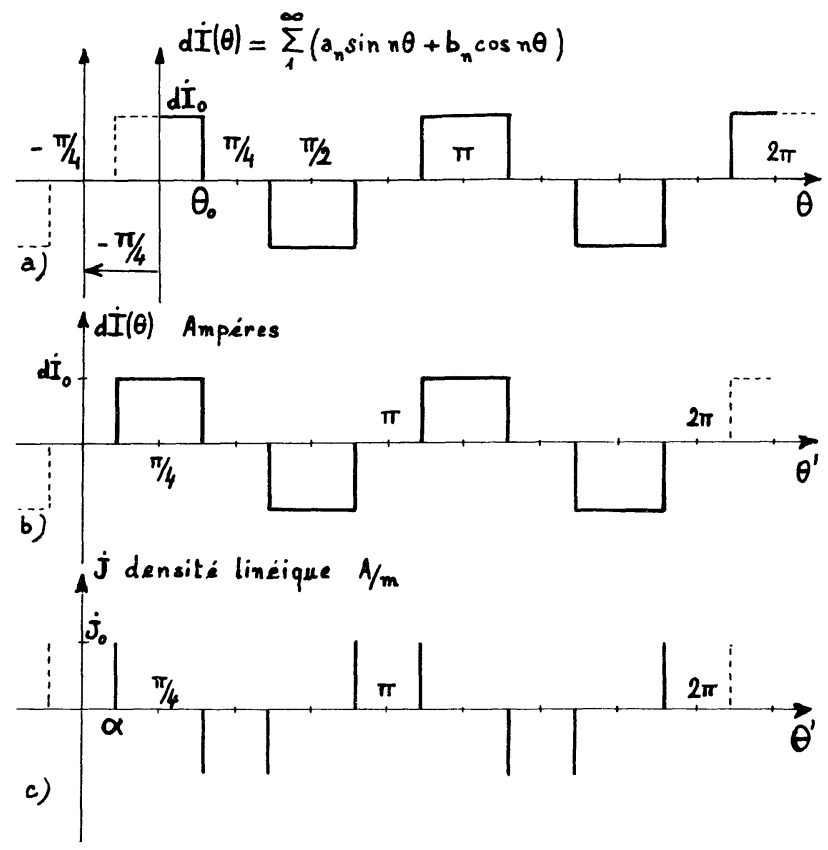

FIG. 8.

L’intensité portée par un petit élément de la figure 7 est donnée par :

$$
\mathrm{d} I_{0}=J_{0} R \mathrm{~d} \alpha .
$$

Sur le cercle de rayon $R$, la variation de l'intensité totale $\mathrm{d} I$ avec $\theta$ est représentée sur la figure $8 \mathrm{~b}$. C'est le développement de cette fonction qui doit être employé dans l'application de la formule (6). Pour atteindre ce développement, considérons tout d'abord la variation représentée sur la figure 8 a. C'est celle déjà rencontrée sur la figure $6 \mathrm{~b}$ et dont nous connaissons le développement, à la réserve près que nous avons maintenant affaire à une couche infiniment mince. Il suffit donc de transposer la formule (13 bis) et on obtient :

$$
\begin{aligned}
\mathrm{d} I(\theta) & =\sum_{k=0}^{\infty} \frac{8 \mathrm{~d} I_{0} \sin n \theta_{0}}{\pi n} \cos n \theta \\
& =\sum_{k=0}^{\infty} \frac{8 J_{0} R \sin n \theta_{0}}{\pi n} \cos n \theta \mathrm{d} \alpha .
\end{aligned}
$$

Opérons tout d'abord une rotation des axes de $-\pi / 4$. On obtient la nouvelle variable $\theta^{\prime}=\theta+\pi / 4$. Puis effectuons le changement de variable défini par $\theta_{0}^{\prime}=\pi / 2-\alpha$.

On obtient :

$\mathrm{d} I\left(\theta^{\prime}\right)$

$$
=\sum_{k=0}^{\infty} \frac{8 R J_{0} \sin [n(\pi / 4-\alpha)]}{\pi n} \cos \left[n\left(\theta^{\prime}-\pi / 4\right)\right] \cdot \mathrm{d} \alpha .
$$


Sachant que $n=2(2 k+1)$, on a :

$$
\mathrm{d} I=+\sum_{k=0}^{\infty} \frac{8 R J_{0} \cos n \alpha}{\pi n} \sin n \theta^{\prime} \mathrm{d} \alpha \text {. }
$$

Par application de (7), on obtient le potentiel complexe créé par 8 petits éléments disposés sur un cercle de rayon $R$ :

$$
\mathrm{d} \mathscr{A}(\mathscr{Z})=\mu_{0} \sum_{k=0}^{\infty} \frac{4 J_{0} \cos n \alpha}{\pi n} \frac{\mathscr{Z}^{n}}{R^{n-1}} \mathrm{~d} \alpha .
$$

Pour vérifier son exactitude, on peut réintégrer cette formule sur un cercle avec la distribution de la formule (9).

Cette formule peut être mise sous une autre forme, en faisant intervenir le courant $\mathrm{d} I_{0}$ porté par chaque élément :

$$
\mathrm{d} \mathscr{A}(\mathscr{Z})=\mu_{0} \sum_{k=0}^{\infty} \frac{4 \mathrm{~d} I_{0} \cos n \alpha}{\pi n} \frac{\mathscr{Z}^{n}}{R^{n}} .
$$

Pour intégrer suivant une courbe différente d'un cercle, on utilisera la densité $J_{0}=\frac{\mathrm{d} I_{0}}{\mathrm{~d} l}, \mathrm{~d} l$ étant l'élément de longueur sur cette courbe d'équation $R=f(\alpha)$. On a :

$$
\mathrm{d} l=\sqrt{R^{2}+R^{\prime 2}} \mathrm{~d} \alpha
$$

$R^{\prime}$ représentant la dérivée de $R$ par rapport à la variable $\alpha$.

Soit :

$$
\mathrm{d} \mathscr{A}(\mathscr{Z})=\mu_{0} \sum_{k=0}^{\infty} \frac{4 J_{0} \sqrt{R^{2}+R^{\prime 2}} \cos n \alpha}{\pi n} \frac{\mathscr{Z}^{n}}{R^{n}} \mathrm{~d} \alpha .
$$

4.2. Champ Quadrupolaire idéal Gréé par 4 NAPPES HYPERBOLIQUES GONJUGUÉES ET ÉQUILATÈRES. - Nous allons montrer que sur les nappes hyperboliques d'équation $R^{2} \cos 2 \alpha=R_{0}^{2}$ la configuration de courant produisant un champ quadrupolaire idéal est donnée par :

$$
J_{0}(R) / J_{0}(0)=R / R_{0}
$$

$J_{0}(0)$ étant la valeur de la densité linéique en $R=R_{0}$.

Calcul de l'élément de longueur $\mathrm{d} l$. - On a :

$$
R^{\prime}=\mathrm{d} R / \mathrm{d} \alpha=R_{0} \sin 2 \alpha / \cos ^{3 / 2} 2 \alpha
$$

d'où l'élément de longueur :

$$
\begin{aligned}
& \mathrm{d} l=\sqrt{R^{2}+R^{\prime 2}} \mathrm{~d} \alpha \\
& \mathrm{d} l=\left(R_{0} / \cos ^{3 / 2} 2 \alpha\right) \mathrm{d} \alpha
\end{aligned}
$$

en reportant dans (15 bis) cette expression de $\mathrm{d} l$ et $(15$ ter $)$, on obtient, avec $R^{n-1}=R_{0}^{n-1} /(\cos 2 \alpha)^{(n-1) / 2}$ :

$\mathrm{d} \mathscr{A}(\mathscr{Z})=\mu_{0} \sum_{k=0}^{\infty} \frac{4 J_{0}(0) \cos n \alpha(\cos 2 \alpha)^{\frac{n-1}{2}}}{\pi n \cos ^{3 / 2} 2 \alpha} \frac{\mathscr{Z}^{n}}{R_{0}^{n-1}} \mathrm{~d} \alpha$

intégrons de 0 à $\pi / 2$, en posant $\varphi=2 \alpha$ :

$\mathscr{A}(\mathscr{Z})$

$=\mu_{0} \sum_{k=0}^{\infty} \int_{0}^{\pi / 2} \frac{4 J_{0}(0)(\cos \varphi)^{2 k-1} \cos (2 k+1) \varphi \mathscr{Z}^{n}}{\pi n R_{0}^{n-1}} \frac{\mathrm{d} \varphi}{2}$ pour $k=0$, les termes en cosinus disparaissent et on obtient :

$$
\begin{aligned}
& \mathscr{A}(\mathscr{Z})=\frac{\mu_{0} J_{0}(0)}{2 R_{0}} \mathscr{Z}^{2} \\
& +\sum_{k=1}^{\infty} \mathscr{Z}^{n} \int_{0}^{\pi / 2} \frac{2 J_{0}(0)(\cos \varphi)^{2 k-1} \cos (2 k+1) \varphi}{\pi n R_{0}^{n-1}} \mathrm{~d} \varphi .
\end{aligned}
$$

Toutes ces intégrales de la forme $\int_{0}^{\pi / 2} \cos ^{\nu-2} x \cos v x \mathrm{~d} x$ sont nulles $\left({ }^{1}\right)$, en conséquence le champ obtenu est purement quadrupolaire, soit :

$$
\mathscr{A}(\mathscr{Z})=\frac{\mu_{0} J_{0}(0)}{2 R_{0}} \mathscr{Z}^{2} .
$$

En conclusion, il a été mis en évidence deux répartitions de courant sur des courbes infiniment minces (cercles et hyperboles équilatères) fournissant une répartition de champ transversal purement quadrupolaire. Sur le cercle, la densité de courant décroît jusqu'à s'annuler quand $\theta$ croît de 0 à $\pi / 4$. Sur l'hyperbole, $J$ croît de $J(0)$ jusqu'à l'infini pour la même variation de $\theta$. On pourrait ainsi penser qu'il existe une courbe intermédiaire - avec direction asymptotique pour $\theta=\pi / 4$ - fournissant le même résultat avec $J=$ constante (d'ou son intérêt). Malheureusement, il ne nous a pas été possible de mettre ce fait en évidence.

Cependant, il ne faut pas oublier que la structure technologiquement la plus intéressante serait celle formée par 4 sections « épaisses » (et non pas des surfaces infiniment minces) et où la densité de courant $j$ serait constante.

Nos efforts ultérieurs ont donc porté sur l'approche de ce problème.

5. Champ produit par 4 sections épaisses. Généralités. - Considérons 4 conducteurs dont les sections sont limitées par 4 courbes $(\mathrm{C})$ identiques et se déduisant les unes des autres par des rotations de centre $\mathrm{O}$ et d'angle $\pi / 2$; soit une couronne d'épaisseur $\mathrm{d} R$ autour du rayon moyen $R$.

Le courant porté par les portions de cette couronne situées dans les bobines peut s'écrire après décomposition en série de Fourier :

$$
\mathrm{d} I(\theta)=\sum_{k=0}^{\infty} A_{n} \sin n \theta \cdot \mathrm{d} R
$$

car pour un choix judicieux de l'origine on peut avoir $B_{n}=0$ et on a vu que :

$$
n=2(2 k+1) \text { avec } k \text { entier } \geqslant 0 \text {. }
$$

On se propose de transférer le courant contenu dans les 4 sections limitées par $(\mathrm{C})$ sur un cercle de rayon $R_{0}$ et produisant dans l'espace utile le même potentiel $\mathscr{A}$.

$\theta_{0}$ étant défini par l'intersection du cercle de rayon $R$

(1) Table of integrals series and products I. S. Gradshteyn et I. M. Ryzhik, Acad. Press, New York, p. 374, no $3631,19$. 


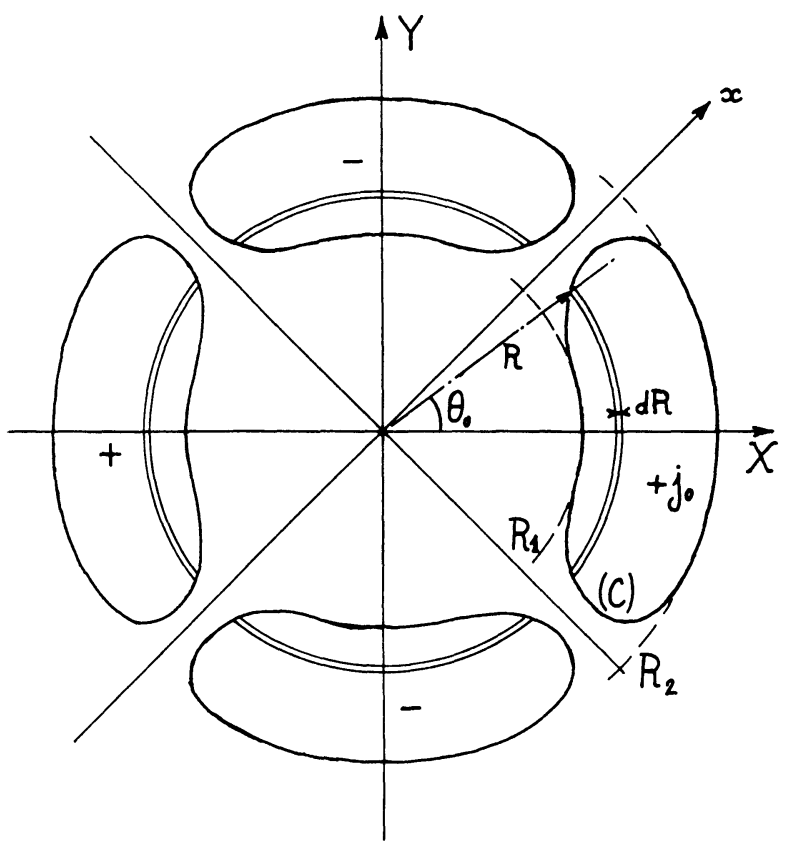

FIG. 9 .

et de la courbe $\mathrm{C}$, l'intensité portée par la couronne est donnée par la formule $(13 \mathrm{ter})$ :

$$
\mathrm{d} I(\theta)=\sum_{k=0}^{\infty} \frac{8 j_{0} \sin n \theta_{0}}{\pi n^{2}} R \mathrm{~d} R \sin n \theta .
$$

La contribution de ce courant au potentiel complexe est :

$$
\mathrm{d} \mathscr{A}(\mathscr{Z})=\frac{\mu_{0}}{2} \sum_{k=0}^{\infty} A_{n} \frac{\mathscr{Z}^{n}}{R^{n}} \mathrm{~d} R
$$

avec $A_{n}$ donné par la formule (13).

Si ce courant disposé sur le cercle de rayon $R$ se trouve ramené sur un cercle déterminé de rayon $R_{0}$ compris par exemple entre $R_{1}$ et $R_{2}$, pour que le potentiel demeure inchangé, on écrira :

$$
\mathrm{d} \mathscr{A}(\mathscr{Z})=\frac{\mu_{0}}{2} \sum_{k=0}^{\infty} \frac{A_{n}\left(R_{0}^{n} / R^{n}\right) \mathscr{Z}^{n}}{R^{n}\left(R_{0}^{n} / R^{n}\right)} \mathrm{d} R .
$$

Il suffit donc de remplacer le coefficient $A_{n}$ par un nouveau coefficient :

$$
\boldsymbol{\alpha}_{n}=A_{n} \frac{R_{0}^{n}}{R^{n}}=\frac{8 j_{0} \sin n \theta_{0}}{\pi n^{2}} \frac{R_{0}^{n}}{R^{n-1}}
$$

avec $\theta_{0}=f(R)$ définissant le contour $\mathrm{C}$.

Alors, on a :

$$
\mathrm{d} \mathscr{A}(\mathscr{Z})=\frac{\mu_{0}}{2} \sum_{k=0}^{\infty} \boldsymbol{\alpha}_{n} \frac{\mathscr{Z}^{n}}{R_{0}^{n}} \mathrm{~d} R .
$$

Le courant transposé du rayon $R$ à $R_{0}$, pour produire le même champ, devient :

$$
\mathrm{d} I(\theta)=\sum_{k=0}^{\infty} \frac{8 j_{0} \sin n \theta_{0}}{\pi n^{2}} \frac{R_{0}^{n}}{R^{n-1}} \mathrm{~d} R \sin n \theta
$$

soit donc une nouvelle densité de courant équivalente :

$$
j_{0}\left(\frac{R_{0}}{R}\right)^{n-1} \quad \text { qui dépend de l'ordre } n \text {. }
$$

Le courant équivalent aux 4 sections limitées par les courbes $(\mathrm{C})$ et ramené au cercle de rayon $R_{0}$ s'obtient en effectuant l'intégration le long de $(\mathrm{C})$ (avec $\theta_{0}=f(R)$ ), soit :

$$
I(\theta)=\int_{R_{1}}^{R_{2}} \sum_{k=0}^{\infty} \frac{8 j_{0} \sin n \theta_{0}}{\pi n^{2}}\left(\frac{R_{0}}{R}\right)^{n-1} \sin n \theta R_{0} \mathrm{~d} R
$$

on obtient :

$$
\begin{aligned}
I(\theta) & =\frac{2 j_{0} R_{0}^{2}}{\pi} \sin 2 \theta \int_{R_{1}}^{R_{2}} \frac{\sin 2 \theta_{0}}{R} \mathrm{~d} R \\
& +\frac{2}{9 \pi} j_{0} R_{0}^{6} \sin 6 \theta \int_{R_{1}}^{R_{2}} \frac{\sin 6 \theta_{0}}{R^{5}} \mathrm{~d} R \\
& +\frac{2}{25 \pi} j_{0} R_{0}^{10} \sin 10 \theta \int_{R_{1}}^{R_{2}} \frac{\sin 10 \theta_{0}}{R^{9}} \mathrm{~d} R \\
& +\frac{2}{49 \pi} j_{0} R_{0}^{14} \sin 14 \theta \int_{R_{1}}^{R_{2}} \frac{\sin 14 \theta_{0}}{R^{13}} \mathrm{~d} R \\
& +\ldots \ldots \ldots \ldots \ldots \ldots \ldots \\
& +\frac{2 j_{0} R_{0}^{n}}{(2 k+1)^{2} \pi} \sin n \theta \int_{R_{1}}^{R_{2}} \frac{\sin n \theta_{0}}{R^{n-1}} \mathrm{~d} R+\ldots
\end{aligned}
$$

Il convient de remarquer que ces formules, difficilement calculables littéralement, se prêtent parfaitement bien au calcul machine.

On a ainsi exprimé directement en courant la contribution au champ quadrupolaire. Les intégrales ainsi mises en évidence ne dépendent que de la géométrie du système; on cherchera à rendre maximum le terme :

$$
\int_{R_{1}}^{R_{2}}\left(\sin 2 \theta_{0} / R\right) \mathrm{d} R \quad \text { (gradient au centre) }
$$

et au contraire à rendre minimum toutes les intégrales d'ordre supérieur (principalement celles en 6, 10 et $14 \theta_{0}$ ) et si possible à annuler celle en $6 \theta_{0}$.

Sur le cercle de rayon $R_{0}$, l'intensité de la forme :

$$
I(\theta)=\sum_{k=0}^{\infty} A_{n} \sin n \theta
$$

est donnée par la formule (16).

A partir de la formule (11), on peut calculer le potentiel produit par cette distribution de courant et obtenir une expression générale :

$$
\begin{aligned}
\mathscr{A}(\mathscr{Z})= & \frac{\mu_{0} j_{0}}{\pi}\left[\mathscr{Z}^{2} \int_{R_{1}}^{R_{2}} \frac{\sin 2 \theta_{0}}{R} \mathrm{~d} R+\frac{\mathscr{Z}^{6}}{9} \int_{R_{1}}^{R_{2}} \frac{\sin 6 \theta_{0}}{R^{5}} \mathrm{~d} R\right. \\
& \left.+\ldots+\frac{\mathscr{Z}^{n}}{(2 k+1)^{2}} \int_{R_{1}}^{R_{2}} \frac{\sin n \theta_{0}}{R^{n-1}} \mathrm{~d} R+\ldots\right]
\end{aligned}
$$


le gradient complexe est donné par :

$$
\begin{aligned}
\mathscr{K}= & \frac{\mathrm{d}^{2} \mathscr{A}}{\mathrm{d} \mathscr{Z}^{2}}=\frac{\mu_{0} j_{0}}{\pi}\left[2 \int_{R_{1}}^{R_{2}} \frac{\sin 2 \theta_{0}}{R} \mathrm{~d} R\right. \\
& +\frac{30}{9} \mathscr{Z}^{4} \int_{R_{1}}^{R_{2}} \frac{\sin 6 \theta_{0}}{R^{5}} \mathrm{~d} R+\ldots \\
& \left.+\frac{n(n-1)}{(2 k+1)^{2}} \mathscr{Z}^{n-2} \int_{R_{1}}^{R_{2}} \frac{\sin n \theta_{0}}{R^{n-1}} \mathrm{~d} R+\ldots\right]
\end{aligned}
$$

si on fait $\theta_{0}=$ const. entre $R_{1}$ et $R_{2}$, l'intégration est aisée et sur l'axe $\mathrm{X}^{\prime} \mathrm{X}(Y=0)$, en appliquant la relation (3), on retrouve la formule (14).

Sur l'axe $\mathrm{x}^{\prime} \mathrm{x}$ ( fig. 5) se déduisant de $\mathrm{X}^{\prime} \mathrm{X}$ par une rotation de $+\pi / 4$, on posera $X=Y=x \sqrt{ } \overline{2}$ et on trouvera la même série que sur l'axe $\mathrm{X}^{\prime} \mathrm{X}$, mais alternée.
Conclusion. - Il ne semble pas qu'il existe de section pour laquelle toutes les intégrales d'ordre supérieur seraient nulles. Cependant, il doit être possible de les rendre très faibles.

Pour cela, il faut se donner au départ une forme de section particulière et étudier systématiquement, en fonction des paramètres définissant cette forme, les conditions les plus favorables pour offrir un champ quadrupolaire aussi parfait que possible et une technologie de construction et bobinage assez simple. C'est ainsi que nous avons procédé [2] avec des sections trapézoïdales qui permettent d'espérer de bons résultats. Une prochaine publication nous permettra de faire l'étude systématique des lentilles à sections trapézoïdales et de comparer résultats expérimentaux et prévisions théoriques quant à la constance du gradient transversal dans l'espace utile.

\section{BIBLIOGRAPHIE}

[1] SePtiER (A.), Les lentilles magnétiques quadrupolaires sans fer, J. Physique, mars 1960, 21, suppl. au no $3,1 \mathrm{~A}-15 \mathrm{~A}$.

[2] Septier (A.), Mouza (J.-C.) et Donadieu (L.), Realization of an ion-free superconducting quadrupole lens, IEEE Trans. on Magnetics, V. MAG-2, 1966, 3, 326-330.

[3] BETH (R. A.), Fields produced by cylindrical current arrays, Brookhaven Nat. Lab., Accelerator Department, Internal Report AADD 102, 1966, March 25.

[4] SePtier (A.), Strong-Focusing Lenses, Advances in Electronics and Electron Physics, 1961, vol. XIV, p. 85-205.

[5] Favereau (M. J.) et Claude (M. I.), Quadrupôles supraconducteurs, Bull. Soc. Française Électriciens, octobre 1964, 58, 658-662.
[6] Kruger (P. G.), SAmpson (W. B.) et Britron (R. B.), A superconducting quadrupole lens, Brookhaven Nat. Lab., Accelerator Department, Internal Report AADD-104-R, 16 septembre 1966.

[7] Schnuriger (J. C.), Création des champs magnétiques utilisés pour la focalisation forte à l'aide de bobines sans fer, $J$. Physique, février 1961, 22, suppl. au no 2, 68 A-69 A.

[8] Asner (A.), DeuTsch (F.) et Iselin (C.), Considerations on supraconducting beam transport elements. C.E.R.N., Internal Report MPS/Int. MA 65-12, 1965.

[9] ASNer (A.) et ISELIN (C.), Some analytical methods for winding configurations of ironless beam transport magnets and lenses, Second International Conference on Magnet Technology (à paraître). 